\title{
DISCLAIMER
}

This report was prepared as an account of work sponsored by an agency of the United States Government. Neither the United States Government nor any agency thereof, nor any of their employees, makes any warranty, express or implied, or assumes any legal liability or responsibility for the accuracy, completeness, or usefulness of any information, apparatus, product, or process disclosed, or represents that its use would not infringe privately owned rights. Reference herein to any specific commercial product, process, or service by trade name, trademark, manufacturer, or otherwise does not necessarily constitute or imply its endorsement, recommendation, or favoring by the United States Government or any agency thereof. The views and opinions of authors expressed herein do not necessarily state or reflect those of the United States Government or any agency thereof.

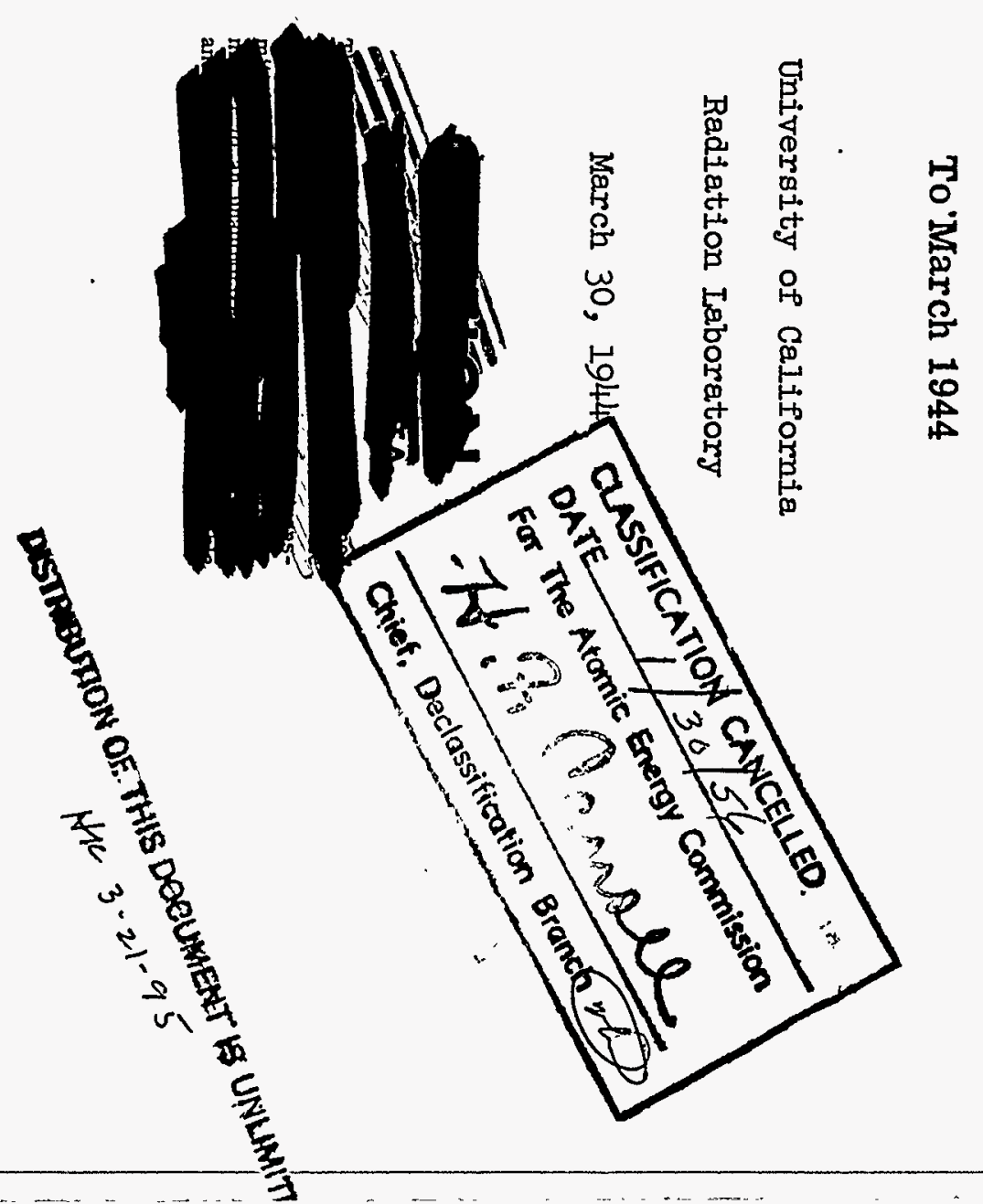




\section{HEALTH AND BIOLOGY}

- Standard Distribution

Argonne National Laboratory

Armed Forces Special Weapons Project

Atomic Energy Commission, Washington

Battelle Memorial Institute

9

Brookhaven National Iaboratory

Bureau of Medicine and Surgery

Carbide and Carbon Chemicals Corporation (K-25 Plant)

Carbide and Carbon Chemicals Corporation ( $\mathrm{Y}-12$ Plant)

Chicago Operations Office

Columbia University (Dunning)

$1-8$

$10-11$

12

$13-16$

17

$18-21$

$22-25$

26

27

Columbia University (Failla)

28

General Electric Company, Richland

35

Iowa State College

$\because$ Knolls Atomic Power Laboratory

Los Alamos

- Massáchusetts Institute of Technology (Kaufmann)

Mound Labóratory

National Advisôry; Committee for Aeronautics

Nationăll Bureau of Stậndarids

Naval Radiological Defense Laboratory

NEPA Project " $i$ " "

New Brunswick Laboratory

New York Operations Office

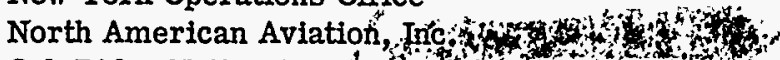

Oak Ridge National Laborator

36

$37-38$

$39-41$

42

$43-45$

46

47

48

49

50

Patent Branch,'Washington ${ }^{3}$. W

67

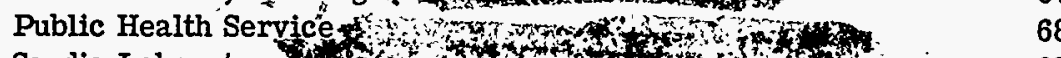

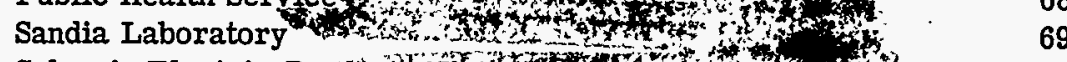

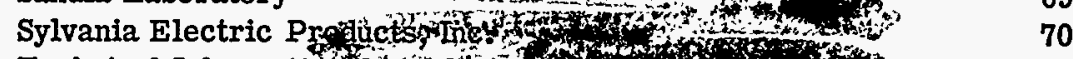

Technical Informatt Branch oR, $71-85$

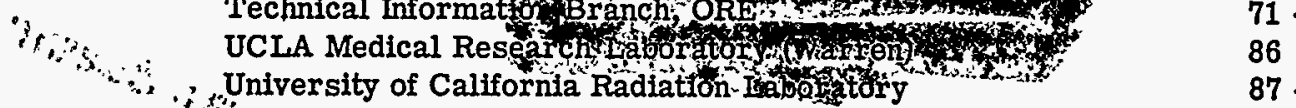

$87-91$

University of Rochester

$92-93$

Univer'sity of Washington

Western Rèserve University (Friedell)

Westinghouse Electric Corporation

Reproduced and distributed by United States Atomic Energy Commission Technical Information Branch, ORE

Oak Ridge, Tennessee

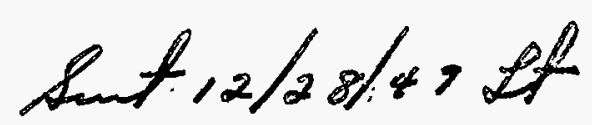

Date Received August 26, 1949

Date Issued September 12, 1949

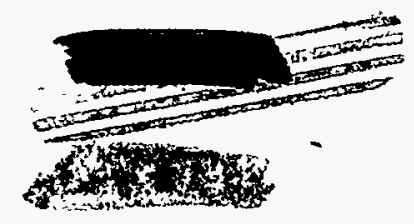




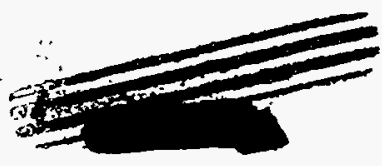

M-4382

\section{A SUMMARY OF THE FISSION PRODUCT METABOLISM STUDIES}

Up to March 1944

The metabolic properties of the carrier free long lived fission products in the adult rat have been investigated at different intervals ranging from one to sixty-eight days following administration of the radioactive material. The radio elements studied include the most important long lived fission products, namely, $\mathrm{Sr}, \mathrm{Y}, \mathrm{Zr}, \mathrm{Cb}, \mathrm{Ru}, \mathrm{I}, \mathrm{Xe}, \mathrm{Cs}, \mathrm{Ba}, \mathrm{La}, \mathrm{Ce}, \mathrm{Pr}, \mathrm{Np}$, and the unseparated uranium free fission mixture. The routes of administration included intraperitoneal, intramuscular, oral and intrapulmonary. The tissues assayed for the distribution of the administered radio elements included heart, liver, kidney, testes, spleen, muscle, skin, stomach, small intestine, large intestine, bone, blood, lungs, brain, fat, adrenals, lymph nodes. Urine and feces were collected separately at daily intervals for assay.

The rats were injected and sacrificed in groups of three for each time interval and the tissues of each group assayed separately and the measured results averaged. All tissue samples were weighed before ashing so as to obtain the distribution per gram wet weight as well as the per organ distribution of the administered radio-element. The entire organ was assayed as a unit for all of the tissues with the exception of muscle, blood, and fat. The entire skin was assayed as a unit in the lung experiments. In the case of bone, the femur and tibia were assayed as well as the remainder of the entire skeleton and the per gram activity of bone as shown in the following table was calculated from the wet weight of the femur and tibia. The ashed samples were all weighed individually so that the proper correction for mass absorption could be made. Each sample was measured at least twice on either an electroscope or Geiger Counter. The per organ data was found to be more consistant than the per gram values and was probably due to variations in both animal size and ratio of the weights of the various organs to the weights of the animals.

The accompanying tables summarize the most pertinent results obtained from these studies. The tissues listed were selected from the original data because of either an initial high uptake or a prolonged retention of the administered radioactive material to such a degree that it is deemed important for the practical consideration of the health hazards of the fission products.

For the fission products which were poorly absorbed from the site of both intramuscular and intraperitoneal injection (namely, $\mathrm{Y}, \mathrm{Zr}, \mathrm{Cb}, \mathrm{Ru}, \mathrm{La}, \mathrm{Ce}, \mathrm{Pr}, \mathrm{Np}$, and the uranium free fission mixture) only the intramuscular data are given here and it is expressed in terms of the distribution of the fraction absorbed from the site of injection. For the radio elements which are completely absorbed following intraperitoneal and intramuscular injection or are absorbed in the digestive tract the distribution is expressed in terms of the administered dose. Due to the difficulty of introducing an exactly known dose into the lungs the distribution in this series of experiments is expressed as distribution of retained activity within the animal. A total of 510 animals were used and 10,500 samples assayed with at least two measurements made on each sample. The average measured recovery of the administered dose in the tissue and excreta was $103.6 \%$.

In addition to these experiments a large series of radio-autographs of lung tissue were made at different times following the intrapulmonary administration of $\mathrm{Y}, \mathrm{Zr}, \mathrm{Cb}, \mathrm{Ru}, \mathrm{Ce}, \mathrm{Np}$, and the fission mixture. Detailed histological studies of the sections and careful examination of the radioautographs under the microscope revealed that these; radio elements were deposited principally in the alveolar structure of the lung. No significint deposition and retention of radioactive material was observed in the blood vessels, bronchial tree, and lymphoid tissliog the lung.

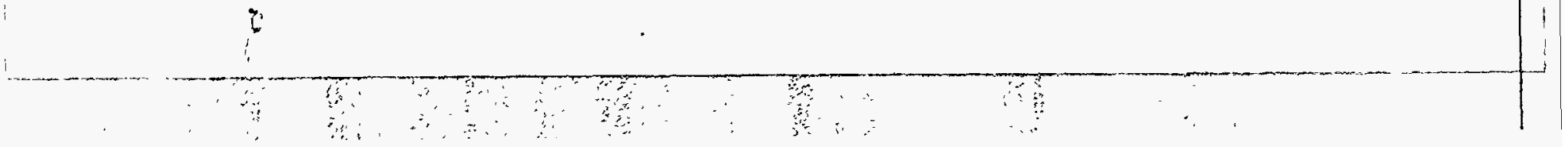




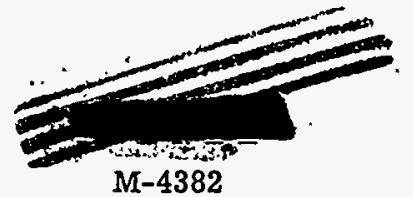

ELEMENT - STRONTIUM (Sr ${ }^{85}$ ) ADMINISTERED IN SOLUTION AS SrCl,

ROUTE OF ADMINISTRATION - INTRAPERITONEAI

Values Indicate Distribution of Administered Activity

Time in Days Following Administration

\begin{tabular}{|c|c|c|c|c|c|c|c|c|}
\hline \multirow[b]{2}{*}{ Tissue } & \multicolumn{2}{|c|}{ One Day } & \multicolumn{2}{|c|}{ Four Days } & \multicolumn{2}{|c|}{ Sixteen Days } & \multicolumn{2}{|c|}{ Sixty-Four Days } \\
\hline & $\begin{array}{l}\text { \% Per } \\
\text { Organ }\end{array}$ & $\begin{array}{l}\text { \% Per. } \\
\text { Gram }\end{array}$ & $\begin{array}{l}\text { \% Per } \\
\text { Organ }\end{array}$ & $\begin{array}{l}\% \text { Per } \\
\text { Gram }\end{array}$ & $\begin{array}{l}\text { \% Per } \\
\text { Organ }\end{array}$ & $\begin{array}{l}\% \text { Per } \\
\text { Gram }\end{array}$ & $\begin{array}{l}\% \text { Per } \\
\text { Organ }\end{array}$ & $\begin{array}{l}\% \text { Per } \\
\text { Gram }\end{array}$ \\
\hline one & 70.2 & 4.7 & 61.7 & 3.8 & 50.4 & 3.3 & 43.3 & 2.1 \\
\hline Muscle & 1.22 & .011 & .36 & .003 & .098 & .0008 & .90 & .006 \\
\hline Skin & .97 & .020 & .45 & .007 & .18 & .004 & .062 & .001 \\
\hline Blood & .32 & .016 & .12 & .004 & .024 & .001 & .008 & .0003 \\
\hline
\end{tabular}

Rate of excretion equivalent to half-time in body of $\sim 200$ days.

\section{ROUTE OF ADMINISTRATION - ORAL} Values Indicate Distribution of Administered Activity (absorption $15 \%$ of

\begin{tabular}{ll}
$\cdot$ & \multicolumn{2}{c}{ One Day } \\
\hline$\%$ Per & $\%$ Per \\
Organ & Gram \\
\hline
\end{tabular}

Bone Muscle Skin

Blood

\begin{tabular}{|c|c|}
\hline \multicolumn{2}{|c|}{ Four Days } \\
\hline $\begin{array}{l}\% \text { Per } \\
\text { Organ }\end{array}$ & $\begin{array}{l}\text { \% Per } \\
\text { Gram }\end{array}$ \\
\hline 9.4 & .71 \\
\hline .40 & .004 \\
\hline .20 & .006 \\
\hline .58 & .033 \\
\hline
\end{tabular}

\begin{tabular}{|c|c|}
\hline \multicolumn{2}{|c|}{ Sixty-Four Days } \\
\hline $\begin{array}{l}\text { \% per } \\
\text { Organ }\end{array}$ & $\begin{array}{l}\% \text { Per } \\
\text { Gram }\end{array}$ \\
\hline 10.9 & .52 \\
\hline .070 & .0005 \\
\hline .032 & .0005 \\
\hline .006 & .0002 \\
\hline
\end{tabular}

Recovery of Administered Dose in Tissues $97.0 \%$

Rate of excretion of absorbed dose equivalent to half time in body of $\sim 200$ days.

\section{ROUTE OF ADMINISTRATION - INTRAPULMONARY}

No significant lung retention.

Total Number of Animals Used . . . . . . . . . . . . 30

Total Number of Tissue Assays . . . . . . . . . . . 750 


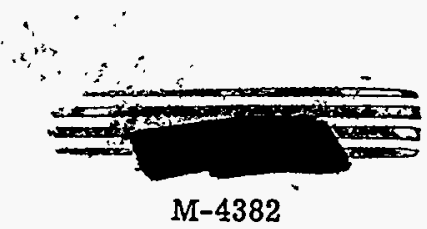

\section{ELEMENT - YTTRIUM $\left(\mathrm{Y}^{88}\right)$ ADMINISTERED IN SOLUTION AS $\mathrm{YCl}_{3}$}

ROUTE OF ADMINISTERED - INTRAMUSCULLAR

Values Indicate Distribution of Absorbed Activity

Time in Days Following Administration

\begin{tabular}{|c|c|c|c|c|c|c|c|c|}
\hline & \multicolumn{2}{|c|}{ One Day } & \multicolumn{2}{|c|}{ Four Days } & \multicolumn{2}{|c|}{ Sixteen Days } & \multicolumn{2}{|c|}{ Sixty-Four Days } \\
\hline & $\begin{array}{l}\% \text { Per } \\
\text { Organ } \\
\end{array}$ & $\begin{array}{l}\% \text { Per } \\
\text { Gram } \\
\end{array}$ & $\begin{array}{l}\% \text { Per } \\
\text { Organ }\end{array}$ & $\begin{array}{l}\% \text { Per } \\
\text { Gram } \\
\end{array}$ & $\begin{array}{l}\% \text { Per } \\
\text { Organ } \\
\end{array}$ & $\begin{array}{l}\% \text { Per } \\
\text { Gram }\end{array}$ & $\begin{array}{l}\% \text { Per } \\
\text { Organ }\end{array}$ & $\begin{array}{l}\% \text { Per } \\
\text { Gram }\end{array}$ \\
\hline Bone & 58.3 & 3.26 & 58.2 & 2.70 & 68.3 & 3.63 & 63.3 & 1.78 \\
\hline Liver & 9.56 & 1.20 & 5.18 & .41 & 1.52 & .14 & .38 & .04 \\
\hline Kidnèy & 2.58 & 1.36 & 2.19 & .82 & 1.84 & .68 & .48 & .20 \\
\hline Spleen & .23 & .31 & .31 & .25 & .30 & .25 & .12 & .15 \\
\hline
\end{tabular}

Recovery of Administered Dose in Tissues 102.1\%

Rate of Excretion Equivalent to Half-Time in Body of $\sim 500$ Days.

ROUTE OF ADMINISTRATION - INTRAPULMONARY

Values Indicate Distribution of Retained Activity

\begin{tabular}{|c|c|c|c|c|c|c|c|c|c|c|}
\hline & \multicolumn{2}{|c|}{ One Day } & \multicolumn{2}{|c|}{ Four Days } & \multicolumn{2}{|c|}{ Twenty-one Days } & \multicolumn{2}{|c|}{ Thirty-two Days } & \multicolumn{2}{|c|}{ Sixty-four Days } \\
\hline & $\begin{array}{l}\% \text { Per } \\
\text { Organ } \\
\end{array}$ & $\begin{array}{l}\% \text { Per } \\
\text { Gram } \\
\end{array}$ & $\begin{array}{l}\% \text { Per } \\
\text { Organ } \\
\end{array}$ & $\begin{array}{l}\% \text { Per } \\
\text { Gram }\end{array}$ & $\begin{array}{l}\% \text { Per } \\
\text { Organ }\end{array}$ & $\begin{array}{l}\% \text { Per } \\
\text { Gram }\end{array}$ & $\begin{array}{l}\% \text { Per } \\
\text { Organ }\end{array}$ & $\begin{array}{l}\% \text { Per } \\
\text { Gram }\end{array}$ & $\begin{array}{l}\% \text { Per } \\
\text { Organ }\end{array}$ & $\begin{array}{l}\% \text { Per } \\
\text { Gram }\end{array}$ \\
\hline & 9.64 & .48 & 39.0 & 2.12 & 72.4 & 3.33 & 85.2 & 3.89 & 83.4 & 3.30 \\
\hline ungs & 82.6 & 34.8 & 45.1 & 23.0 & 16.9 & 8.79 & 2.67 & 1.60 & 6.44 & 2.85 \\
\hline & .95 & .074 & 3.21 & .26 & 1.47 & .13 & .78 & .051 & .42 & .029 \\
\hline & .56 & .22 & 2.03 & .73 & .99 & .37 & 1.20 & .39 & .50 & .16 \\
\hline pleen & .054 & .050 & .14 & .13 & .16 & .16 & .28 & .19 & .20 & .18 \\
\hline
\end{tabular}

ROUTE OF ADMINLSTRATION - ORAL

Absorption from Digestive Tract $<0.2 \%$

Total Number of Animals Used ................. 66

Total Number of Tissue Assays . . . . . . . . . . . . 1,380

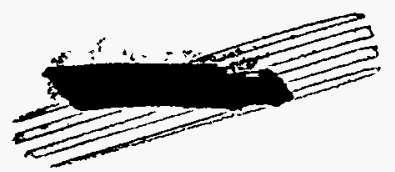




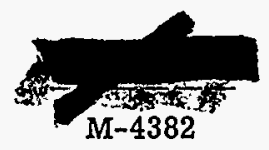

ELEMENT - ZIRCONIUM $\left(\mathrm{Zr}^{89}, \mathrm{Zr}^{93}\right)$ ADMINISTERED AS A SOLUTION OF ZrOCl2

\section{ROUTE OF ADMINISTRATION - INTRAMUSCULAR}

Values Indicate Distribution of Absorbed Activity

Time in Days Following Administrátion

\begin{tabular}{|c|c|c|c|c|c|c|c|c|}
\hline \multirow[b]{2}{*}{ Tissue } & \multicolumn{2}{|c|}{ One Day } & \multicolumn{2}{|c|}{ Four Days } & \multicolumn{2}{|c|}{ Sixteen Days } & \multicolumn{2}{|c|}{ Thirty-Two Days } \\
\hline & $\begin{array}{l}\% \text { Per } \\
\text { Organ }\end{array}$ & $\begin{array}{l}\% \text { Per } \\
\text { Gram }\end{array}$ & $\begin{array}{l}\text { \% Per } \\
\text { Organ }\end{array}$ & $\begin{array}{l}\% \text { Per } \\
\text { Gram }\end{array}$ & $\begin{array}{l}\text { \% Per } \\
\text { Organ }\end{array}$ & $\begin{array}{l}\% \text { Per } \\
\text { Gram }\end{array}$ & $\begin{array}{l}\% \text { Per } \\
\text { Organ }\end{array}$ & $\begin{array}{l}\% \text { Per } \\
\text { Gram }\end{array}$ \\
\hline Bone & 2.38 & .15 & 38.8 & 2.02 & 57.8 & 2.04 & 38.2 & 2.14 \\
\hline Liver & 1.96 & .17 & 4.51 & .65 & 3.73 & .35 & 2.37 & .23 \\
\hline Kidney & .35 & .19 & 2.63 & 1.36 & 1.60 & .63 & 1.56 & .67 \\
\hline Spleen & .051 & .089 & .62 & .54 & .49 & .53 & .25 & .32 \\
\hline
\end{tabular}

Recovery of Administered Dose in Tissues 104.4\%

Rate of Excretion Equivalent to Half-Time in Body $\sim 80$ Days

\section{ROUTE OF ADMINLSTRATION - INTRAPULMONARY}

Values Indicate Distribution of Retained Activity

\begin{tabular}{|c|c|c|c|c|c|c|c|c|}
\hline & \multicolumn{2}{|c|}{ One Day } & \multicolumn{2}{|c|}{ Four Days } & \multicolumn{2}{|c|}{ Thirty-Two Days } & \multicolumn{2}{|c|}{ Sixty-Four Days } \\
\hline & $\begin{array}{l}\% \text { Per } \\
\text { Organ }\end{array}$ & $\begin{array}{l}\% \text { Per } \\
\text { Gram }\end{array}$ & $\begin{array}{l}\% \text { Per } \\
\text { Organ }\end{array}$ & $\begin{array}{l}\% \text { Per } \\
\text { Gram }\end{array}$ & $\begin{array}{l}\text { \% Per } \\
\text { Organ }\end{array}$ & $\begin{array}{l}\% \text { Per } \\
\text { Gram }\end{array}$ & $\begin{array}{l}\text { \% Per } \\
\text { Organ }\end{array}$ & $\begin{array}{l}\% \text { Per } \\
\text { Gram }\end{array}$ \\
\hline Bone & 10.2 & .50 & 3.85 & .21 & 16.3 & 1.08 & 16.3 & .98 \\
\hline Lung & 77.9 & 54.4 & 92.2 & 50.3 & 75.9 & 53.0 & 62.0 & 31.0 \\
\hline Liver & .82 & .06 & .36 & .03 & .50 & .04 & 1.14 & .12 \\
\hline Kidney & .13 & .06 & .20 & .08 & .29 & .12 & .60 & .25 \\
\hline Spleen & .10 & .10 & .27 & .38 & .36 & .50 & .65 & .96 \\
\hline
\end{tabular}

\section{ROUTE OF ADMINISTRATION - ORAL}

Absorption from Digestive Tract $<0.01 \%$,

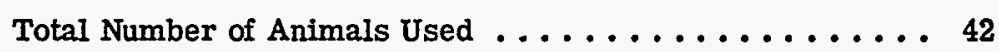

Total Number of Tissue Assays . . . . . . . . . . . . 900

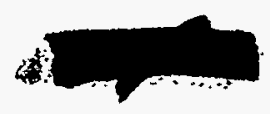




\section{ELEMENT - COLUMBIUM (Cb ${ }^{93}$ ) ADMINISTERED IN SOLUTION AS CbOCl 3}

ROUTE OF ADMINISTRATION - INTRAMUSCULAR

Values Indicate Distribution of Absorbed Activity

Time in Days Following Administration

\begin{tabular}{|c|c|c|c|c|c|c|c|c|}
\hline & \multicolumn{2}{|c|}{ One Day } & \multicolumn{2}{|c|}{ Four Days } & \multicolumn{2}{|c|}{ Sixteen Days } & \multicolumn{2}{|c|}{ Sixty-Eight Days } \\
\hline & $\begin{array}{l}\text { \% Per } \\
\text { Organ }\end{array}$ & $\begin{array}{l}\% \text { Per } \\
\text { Gram }\end{array}$ & $\begin{array}{l}\text { \% Per } \\
\text { Organ }\end{array}$ & $\begin{array}{l}\% \text { Per } \\
\text { Gram }\end{array}$ & $\begin{array}{l}\text { \% Per } \\
\text { Organ }\end{array}$ & $\begin{array}{l}\% \text { Per } \\
\text { Gram }\end{array}$ & $\begin{array}{l}\text { \% Per } \\
\text { Organ }\end{array}$ & $\begin{array}{l}\% \text { Per } \\
\text { Gram }\end{array}$ \\
\hline Bone & 38.4 & 1.48 & 35.5 & 1.74 & 22.6 & 1.94 & 11.0 & .85 \\
\hline Liver & 8.91 & 1.40 & 7.29 & 1.47 & 4.54 & .63 & 1.94 & .29 \\
\hline Kidney & 1.35 & .92 & 1.54 & 1.35 & 1.54 & .93 & .93 & .61 \\
\hline Spleen & .59 & .78 & .76 & 1.24 & .66 & .86 & .34 & .65 \\
\hline Blood & 25.0 & 1.91 & 5.04 & .50 & 1.33 & .093 & .39 & .033 \\
\hline
\end{tabular}

Recovery of Administered Dose in Tissues $102.5 \%$

Rate of Excretion Equivalent to Half-Time in the Body $\sim 50$ Days

ROUTE OF ADMINISTRATION - INTRAPULMONARY

Values Indicate Distribution of Retained Activity

\begin{tabular}{|c|c|c|c|c|c|c|}
\hline & \multicolumn{2}{|c|}{ One Day } & \multicolumn{2}{|c|}{ Four Days } & \multicolumn{2}{|c|}{ Sixteen Days } \\
\hline & $\begin{array}{l}\text { \% Per } \\
\text { Organ }\end{array}$ & $\begin{array}{l}\% \text { Per } \\
\text { Gram }\end{array}$ & $\begin{array}{l}\% \text { Per } \\
\text { Organ }\end{array}$ & $\begin{array}{c}\% \text { Per } \\
\text { Gram }\end{array}$ & $\begin{array}{l}\% \text { Per } \\
\text { Organ }\end{array}$ & $\begin{array}{c}\% \text { Per } \\
\text { Gram }\end{array}$ \\
\hline Bone & 6.75 & .036 & 16.5 & .89 & 18.9 & 1.36 \\
\hline Lung & 75.7 & 62.1 & 58.3 & 50.4 & 51.3 & 40.9 \\
\hline Liver & 1.02 & .14 & 1.94 & .29 & 4.03 & .49 \\
\hline Kidney & .29 & .17 & .58 & .40 & 1.38 & 1.00 \\
\hline Spleen & .05 & .10 & .23 & .34 & .54 & .82 \\
\hline
\end{tabular}

\section{ROUTE OF ADMINISTRATION - ORAL}

Absorption from Digestive Tract $<1 . \%$

Total Number of Animals Used . . . . . . . . . . . . . 51

Total Number of Tissue Assays . . . . . . . . . . . . . . 800

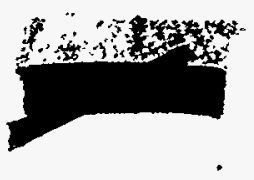


ELEMENT - RUTHENIUM (Ru >104) ADMLNISTERED AS A SOLUTION OF RuCl4

\section{ROUTE OF ADMINISTRATION - INTRAMUSCULAR}

Values Indicate Distribution of Absorbed Activity

Time in Days Following Administration

\begin{tabular}{|c|c|c|c|c|c|c|}
\hline \multirow[t]{2}{*}{ 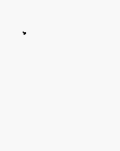 } & \multicolumn{2}{|c|}{ Four Days } & \multicolumn{2}{|c|}{ Sixteen Days } & \multicolumn{2}{|c|}{ Sixty-four Days } \\
\hline & $\begin{array}{l}\% \text { Per } \\
\text { Organ }\end{array}$ & $\begin{array}{c}\% \text { Per } \\
\text { Gram }\end{array}$ & $\begin{array}{l}\% \text { Per } \\
\text { Organ }\end{array}$ & $\begin{array}{l}\% \text { Per } \\
\text { Gram }\end{array}$ & $\begin{array}{l}\% \text { Per } \\
\text { Organ }\end{array}$ & $\begin{array}{c}\% \text { Per } \\
\text { Gram }\end{array}$ \\
\hline Bone & 10.2 & .29 & 5.32 & .16 & 3.52 & .051 \\
\hline Liver & 2.88 & .24 & 1.26 & .13 & .27 & .015 \\
\hline Kidney & 3.48 & 1.36 & 1.41 & .67 & .46 & .14 \\
\hline Spleen & .44 & .36 & .33 & .30 & .068 & .049 \\
\hline
\end{tabular}

Recovery of Administered Dose in Tissues 102.9\%

Rate of Excretion is Equivalent to Half-Time in Body $\sim 20$ Days.

\section{ROUTE OF ADMINLSTRATION - INTRAPULMONARY}

Values Indicate Distribution of Retained Activity

\begin{tabular}{|c|c|c|c|c|c|c|c|c|}
\hline & \multicolumn{2}{|c|}{ Four Days } & \multicolumn{2}{|c|}{ Sixteen Days } & \multicolumn{2}{|c|}{ Thirty-Two Days } & \multicolumn{2}{|c|}{ Sixty-Four Days } \\
\hline & $\begin{array}{l}\% \text { Per } \\
\text { Organ }\end{array}$ & $\begin{array}{l}\% \text { Per } \\
\text { Gram }\end{array}$ & $\begin{array}{l}\% \text { Per } \\
\text { Organ }\end{array}$ & $\begin{array}{l}\% \text { Per } \\
\text { Gram }\end{array}$ & $\begin{array}{l}\% \text { Per } \\
\text { Organ }\end{array}$ & $\begin{array}{l}\% \text { Per } \\
\text { Gram }\end{array}$ & $\begin{array}{l}\% \text { Per } \\
\text { Organ }\end{array}$ & $\begin{array}{l}\% \text { Per } \\
\text { Gram }\end{array}$ \\
\hline Bone & 2.45 & 13 & 7.42 & .37 & 7.29 & .35 & 7.34 & .30 \\
\hline Lungs & 63.6 & 23.6 & 58.4 & 20.8 & 50.3 & 21.0 & 25.5 & 12.6 \\
\hline Liver & 3.20 & .20 & 2.85 & .22 & 3.17 & .25 & 2.65 & .17 \\
\hline Kidney & 4.73 & 1.36 & 7.59 & 2.38 & 4.01 & 1.37 & 4.47 & 1.41 \\
\hline Spleen & .70 & .47 & .75 & .42 & .54 & .45 & .43 & .34 \\
\hline
\end{tabular}

\section{ROUTE OF ADMINISTRATION - ORAL}

Absorption from Digestive Tract $<0.01 \%$

Total Number of Animals Used . . . . . . . . . . . . . 36

Total Number of Tissue Assays . . . . . . . . . . . . 800

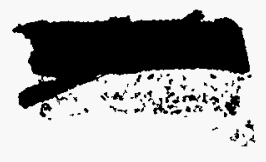


ELEMENT - IODINE (I ${ }^{131}$ ) ADMINISTERED AS A SOLUTION OF NaI. (HUMAN STUDIES)

ROUTE OF ADMINLSTRATION - ORAL

Absorption by way of the Digestive Tract $100 \%$

Retention by Thyroid Gland of Normal Human Adults $20 \%$

Rate of Excretion Exclusive of that in the Thyroid Equivalent to

Half-Time in Body <2 Days.

Rate of Loss from the Thyroid Equivalent to Half-Time in the Gland $>30$ Days.

\section{ELEMENT - XENON (Xe ${ }^{127}$ ) ADMINISTERED AS THE GAS}

\section{ROUTE OF ADMINISTRATION - INHALATION}

Distribution at equilibrium proportional to Fat Content of Tissues Ranging from 2.2 in Fat to 0.1 for Blood of the Concentration of $\mathrm{Xe} 127$ in the Inhaled Air.

Human Studies with $\mathrm{Xe}^{127}$ Done Elsewhere Indicate a Half-Period for equilibrium for either Absorption or Excretion to be $\sim 1$ Hours.

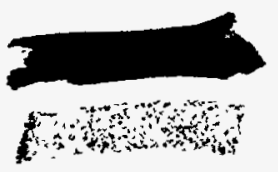




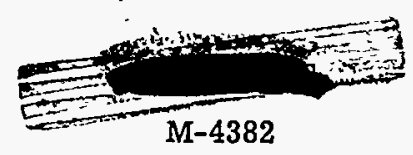

ELEMENT - CESIUM (Cs ${ }^{135}$ ) ADMINISTERED IN SOLUTION AS CSCl

\section{ROUTE OF ADMINISTRATION - INTRAMUSCULAR}

Values Indicate Distribution of Administered Activity - Time in Days Following Administration

\begin{tabular}{|c|c|c|c|c|c|c|c|c|}
\hline \multirow[b]{2}{*}{ Tissue } & \multicolumn{2}{|c|}{ One Day } & \multicolumn{2}{|c|}{ Four Days } & \multicolumn{2}{|c|}{ Sixteen Days } & \multicolumn{2}{|c|}{ Sixty-Eight Days } \\
\hline & $\begin{array}{l}\% \text { Per } \\
\text { Organ }\end{array}$ & $\begin{array}{l}\% \text { Per } \\
\text { Gram }\end{array}$ & $\begin{array}{l}\% \text { Per } \\
\text { Organ }\end{array}$ & $\begin{array}{l}\% \text { Per } \\
\text { Gram }\end{array}$ & $\begin{array}{l}\% \text { Per } \\
\text { Organ }\end{array}$ & $\begin{array}{l}\% \text { Per } \\
\text { Gram }\end{array}$ & $\begin{array}{l}\% \text { Per } \\
\text { Organ }\end{array}$ & $\begin{array}{l}\% \text { Per } \\
\text { Gram }\end{array}$ \\
\hline Bone & 2.60 & .35 & 3.02 & .22 & 2.64 & .14 & .30 & .017 \\
\hline Liver & 5.72 & .60 & 1.94 & .23 & .84 & .10 & .028 & .0063 \\
\hline Kidney & 1.03 & .53 & .43 & .28 & .29 & .16 & .010 & .005 \\
\hline Spleen & .42 & .46 & .12 & .13 & .067 & .11 & .005 & .009 \\
\hline Muscle & 36.3 & .48 & 29.8 & .39 & 27.0 & .31 & 1.89 & .018 \\
\hline
\end{tabular}

Rate of Excretion Equivalent to Half-Time in the Body of $\sim 10$ Days

$$
\text { ROUTE OF ADMINISTRATION - ORAL } \begin{gathered}
\text { (Absorption 100\% of } \\
\text { Administered Dose) }
\end{gathered}
$$

Values Indicate Distribution of Administered Activity

\begin{tabular}{|c|c|c|c|c|c|c|c|c|}
\hline & \multicolumn{2}{|c|}{ One Day } & \multicolumn{2}{|c|}{ Four Days } & \multicolumn{2}{|c|}{ Sixteen Days } & \multicolumn{2}{|c|}{ Sixty-Eight Days } \\
\hline & $\begin{array}{l}\% \text { Per } \\
\text { Organ } \\
\end{array}$ & $\begin{array}{l}\% \text { Per } \\
\text { Gram }\end{array}$ & $\begin{array}{l}\% \text { Per } \\
\text { Organ }\end{array}$ & $\begin{array}{l}\% \text { Per } \\
\text { Gram } \\
\end{array}$ & $\begin{array}{l}\% \text { Per } \\
\text { Organ }\end{array}$ & $\begin{array}{l}\% \text { Per } \\
\text { Gram }\end{array}$ & $\begin{array}{l}\% \text { Per } \\
\text { Organ }\end{array}$ & $\begin{array}{l}\% \text { Per } \\
\text { Gram }\end{array}$ \\
\hline Bone & 7.37 & .43 & 3.46 & .17 & 1.59 & .091 & .51 & .0030 \\
\hline Liver & 4.14 & .58 & 2.04 & .20 & .79 & .089 & .035 & .0046 \\
\hline Kidney & 2.08 & 1.14 & .48 & .23 & .17 & .095 & .0063 & .0040 \\
\hline Spleen & .54 & .66 & .10 & .10 & .041 & .062 & .005 & .008 \\
\hline Muscle & 42.6 & .54 & 19.6 & .26 & 23.1 & .27 & 1.58 & .020 \\
\hline
\end{tabular}

Average Recovery in Tissues of Administered Dose 95.4\%

Rate of Excretion Equivalent to Half-Time in Body of $\sim 10$ Days

\section{ROUTE OF ADMINISTRATION - INTRAPULMONARY}

No Significant Lung Retention Noted.

Total Number of Animals Used $\ldots \ldots \ldots \ldots \ldots \ldots \ldots$

Total Number of Tissue Assays

Hot

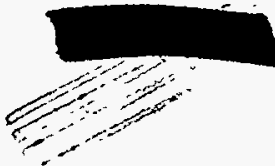




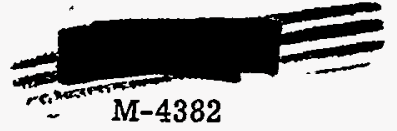

ELEMENT - BARTUM $\left(\mathrm{Ba}^{133}, \mathrm{Ba}^{140}\right)$ ADMINISTERED AS A SOLUTION OF $\mathrm{BaCl}_{2}$

ROUTES OF ADMINLSTRATION - INTRAMUSCULAR AND INTRAPERITONEAL

Values Indicate Distribution of Administered Activity

Time in Days Following Administration

\begin{tabular}{|c|c|c|c|c|c|c|c|c|c|c|}
\hline \multirow[b]{2}{*}{ Tissue } & \multicolumn{2}{|c|}{ One Day } & \multicolumn{2}{|c|}{ Four Days } & \multicolumn{2}{|c|}{ Eight Days } & \multicolumn{2}{|c|}{ Sixteen Days } & \multicolumn{2}{|c|}{ Thirty-Two Days } \\
\hline & $\begin{array}{l}\% \text { Per } \\
\text { Organ } \\
\end{array}$ & $\begin{array}{l}\text { \% Per } \\
\text { Gram }\end{array}$ & $\begin{array}{l}\% \text { Per } \\
\text { Organ }\end{array}$ & $\begin{array}{l}\% \text { Per } \\
\text { Gram }\end{array}$ & $\begin{array}{l}\% \text { Per } \\
\text { Organ }\end{array}$ & $\begin{array}{l}\% \text { Per } \\
\text { Gram }\end{array}$ & $\begin{array}{l}\% \text { Per } \\
\text { Organ }\end{array}$ & $\begin{array}{l}\% \text { Per } \\
\text { Gram }\end{array}$ & $\begin{array}{l}\% \text { Per } \\
\text { Organ }\end{array}$ & $\begin{array}{c}\% \text { Per } \\
\text { Gram }\end{array}$ \\
\hline Bone & 54.2 & 4.39 & 59.6 & 3.68 & 60.3 & 4.96 & 59.5 & 3.26 & 49.1 & 3.51 \\
\hline Muscle & .25 & .0033 & .12 & .0007 & .56 & .0065 & .28 & .0006 & $<.2$ & $<.0002$ \\
\hline Skin & .59 & .015 & .51 & .0093 & .26 & .0072 & .17 & .0028 & .090 & .0025 \\
\hline Blood & .10 & .0063 & .036 & .0017 & .022 & .0013 & .022 & .0009 & $<.01$ & $<.0005$ \\
\hline
\end{tabular}

\section{ROUTE OF ADMINISTRATION - ORAL}

Values Indicate Distribution of Administered Activity $\begin{gathered}\text { (Absorption 10\% of } \\ \text { administered dose) }\end{gathered}$

\begin{tabular}{|c|c|c|c|c|c|c|c|c|c|}
\hline \multicolumn{2}{|c|}{ One Day } & \multicolumn{2}{|c|}{ Four Days } & \multicolumn{2}{|c|}{ Eight Days } & \multicolumn{2}{|c|}{ Sixteen Days } & \multicolumn{2}{|c|}{ Thirty-Two Days } \\
\hline $\begin{array}{l}\% \text { Per } \\
\text { Organ }\end{array}$ & $\begin{array}{l}\% \text { Per } \\
\text { Gram }\end{array}$ & $\begin{array}{l}\text { \% Per } \\
\text { Organ }\end{array}$ & $\begin{array}{l}\% \text { Per } \\
\text { Gram }\end{array}$ & $\begin{array}{l}\% \text { Per } \\
\text { Organ }\end{array}$ & $\begin{array}{l}\% \text { Per } \\
\text { Gram }\end{array}$ & $\begin{array}{l}\% \text { Per } \\
\text { Organ }\end{array}$ & $\begin{array}{l}\% \text { Per } \\
\text { Gram }\end{array}$ & $\begin{array}{l}\text { \% Per } \\
\text { Organ }\end{array}$ & $\begin{array}{l}\% \text { Per } \\
\text { Gram }\end{array}$ \\
\hline 8.87 & .31 & 4.51 & .38 & 11.3 & .66 & 9.82 & .76 & 3.80 & .29 \\
\hline .049 & .0005 & .022 & .0002 & .54 & .0058 & .02 & .0002 & $<.04$ & $<.0004$ \\
\hline .29 & .007 & .085 & .0008 & .070 & .0028 & .092 & .0008 & $<.04$ & $<.001$ \\
\hline .009 & .001 & .006 & .0004 & .015 & .0009 & .003 & .0003 & $<.006$ & $<.0006$ \\
\hline
\end{tabular}

Average Recovery in Tissues of Administered Dose $107.5 \%$

Rate of Excretion of Retained Activity for Both Routes of Administration Equivalent to Half-Time in Body 200 Days.

Total Number of Animals Used ............... 57

. Total Number of Tissue Assays . . . . . . . . . . . 1,250

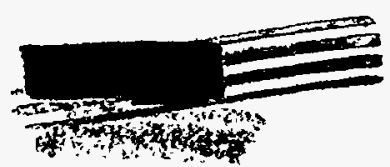




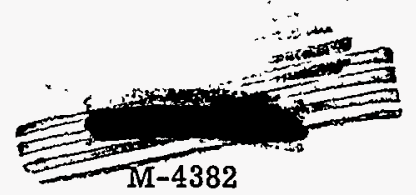

\section{ELEMENT - LANTHANUM (La ${ }^{140}$ ) ADMINISTERED AS A SOLUTION OF $\mathrm{LaCl}_{3}$}

\section{ROUTE OF ADMINISTRATION - INTRAMUSCULAR}

Values Indicate Distribution of Absorbed Activity

Time in Days Following Administration

\begin{tabular}{|c|c|c|c|c|c|c|}
\hline \multirow[b]{2}{*}{ Tissue } & \multicolumn{2}{|c|}{ One Day } & \multicolumn{2}{|c|}{ Four Days } & \multicolumn{2}{|c|}{ Eight Days } \\
\hline & $\begin{array}{l}\% \text { Per } \\
\text { Organ }\end{array}$ & $\begin{array}{l}\% \text { Per } \\
\text { Gram }\end{array}$ & $\begin{array}{l}\% \text { Per } \\
\text { Organ }\end{array}$ & $\begin{array}{l}\% \text { Per } \\
\text { Gram }\end{array}$ & $\begin{array}{l}\% \text { Per } \\
\text { Organ }\end{array}$ & $\begin{array}{l}\% \text { Per } \\
\text { Gram }\end{array}$ \\
\hline Bone & 25.9 & .91 & 42.4 & 1.48 & 30.5 & 1.36 \\
\hline Liver & 69.8 & 6.61 & 48.4 & 4.31 & 23.2 & 2.26 \\
\hline Kidney & 1.72 & .95 & 1.60 & .79 & 1.04 & .48 \\
\hline Spleen & .18 & .29 & .12 & .11 & .11 & .13 \\
\hline
\end{tabular}

Recovery of Administered Dose in Tissues 105.2\%

Excretion Rate Equivalent to Half-Time in Body >16 Days

\section{ROUTE OF ADMINISTRATION - INTRAPULMONARY}

Values Indicate Distribution of Retained Activity

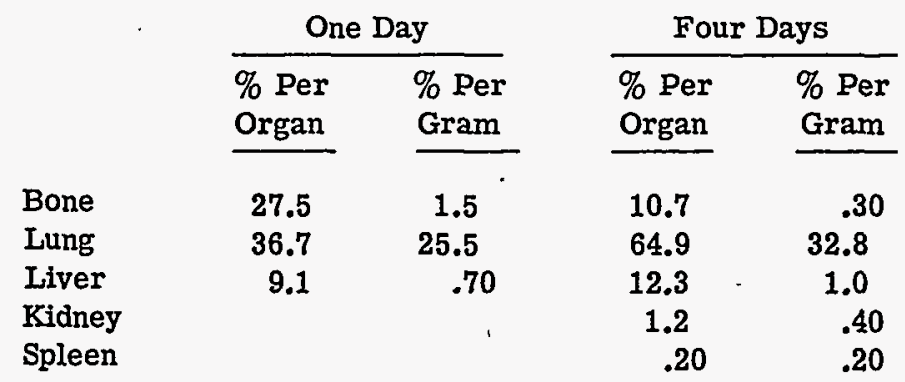

\section{ROUTE OF ADMINISTRATION - ORAL}

Absorption from the Digestive Tract $<0.3 \%$

Total Number of Animals Used . . . . . . . . . . . . . . . 25

Total Number of Tissues Assayed ............ 400

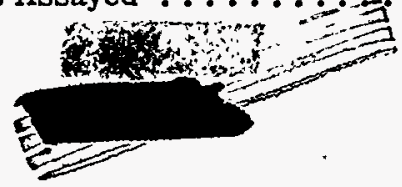


ELEMENT - CERIUM (Ce ${ }^{>140}$ ) ADMINISTERED AS A SOLUTION OF $\mathrm{CeCl}_{3}$

\section{ROUTE OF ADMINISTRATION - INTRAMUSCULAR}

Values Indicate Distribution of Absorbed Activity

Time in Days Following Administration

\begin{tabular}{|c|c|c|c|c|c|c|c|c|}
\hline & \multicolumn{2}{|c|}{ One Day } & \multicolumn{2}{|c|}{ Four Days } & \multicolumn{2}{|c|}{ Sixteen Days } & \multicolumn{2}{|c|}{ Sixty-Four Days } \\
\hline & $\begin{array}{l}\% \text { Per } \\
\text { Organ }\end{array}$ & $\begin{array}{l}\% \text { Per } \\
\text { Gram }\end{array}$ & $\begin{array}{l}\% \text { Per } \\
\text { Organ }\end{array}$ & $\begin{array}{l}\% \text { Per } \\
\text { Gram }\end{array}$ & $\begin{array}{l}\text { \% Per } \\
\text { Organ }\end{array}$ & $\begin{array}{l}\% \text { Per } \\
\text { Gram }\end{array}$ & $\begin{array}{l}\text { \% Per } \\
\text { Organ }\end{array}$ & $\begin{array}{l}\% \text { Per } \\
\text { Gram }\end{array}$ \\
\hline Bone & 34.9 & 1.82 & 37.2 & 1.56 & 34.7 & 1.80 & 27.3 & 1.83 \\
\hline Liver & 41.2 & 5.72 & 42.5 & 7.15 & 13.9 & 1.71 & 2.72 & .51 \\
\hline Kidney & 3.61 & 1.86 & 2.78 & 1.61 & 1.31 & .72 & .28 & .22 \\
\hline Spleen & .42 & .51 & .27 & .42 & .21 & .27 & .15 & .34 \\
\hline
\end{tabular}

Recovery of Administered Dose in Tissues $100.2 \%$

Excretion Rate Equivalent to Half-Time in Body $\sim 50$ Days.

ROUTE OF ADMINISTRATION - INTRAPULMONARY

Values Indicate Distribution of Retained Activity

\begin{tabular}{|c|c|c|c|c|c|c|c|c|}
\hline & \multicolumn{2}{|c|}{ One Day } & \multicolumn{2}{|c|}{ Four Days } & \multicolumn{2}{|c|}{ Sixteen Days } & \multicolumn{2}{|c|}{ Sixty-Four Days } \\
\hline & $\begin{array}{l}\% \text { Per } \\
\text { Organ }\end{array}$ & $\begin{array}{l}\% \text { Per } \\
\text { Gram }\end{array}$ & $\begin{array}{l}\% \text { Per } \\
\text { Organ }\end{array}$ & $\begin{array}{l}\% \text { Per } \\
\text { Gram }\end{array}$ & $\begin{array}{l}\% \text { Per } \\
\text { Organ }\end{array}$ & $\begin{array}{l}\text { \% Per } \\
\text { Gram }\end{array}$ & $\begin{array}{l}\% \text { Per } \\
\text { Organ }\end{array}$ & $\begin{array}{l}\text { \% Per } \\
\text { Gram }\end{array}$ \\
\hline Bone & 14.0 & .55 & 21.2 & 1.53 & 37.6 & 2.40 & 74.6 & 4.49 \\
\hline Lungs & 66.5 & 52.7 & 49.5 & 29.3 & 35.3 & 21.6 & 9.43 & 8.76 \\
\hline Liver & 14.6 & 1.73 & 16.3 & 1.66 & 18.2 & 2.53 & 7.04 & 1.43 \\
\hline Kidney & .58 & .35 & 1.70 & .92 & .77 & .47 & .66 & .53 \\
\hline Spleen & .048 & .071 & .080 & .12 & .17 & .27 & .20 & .50 \\
\hline
\end{tabular}

\section{ROUTE OF ADMINISTRATION - ORAI}

Absorption from the Digestive Tract $<0.05 \%$

Total Number of Animals Used $\ldots . . . . . . . . . . . .51$

Total Number of Tissues Assayed . . . . . . . . . . . . 1,100

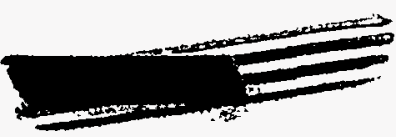


ELEMENT - PRASEODYMIUM $\left(\mathrm{Pr}^{143}\right)$ ADMTNISTERED AS A SOLUTION OF $\mathrm{PrCl}_{3}$

ROUTE OF ADMINISTRATION - INTRAMUSCULAR

Values Indicate Distribution of Absorbed Activity

Time in Days Following Administration

\begin{tabular}{|c|c|c|c|c|c|c|c|c|}
\hline \multirow[b]{2}{*}{ Tissue } & \multicolumn{2}{|c|}{ One Day } & \multicolumn{2}{|c|}{ Four Days } & \multicolumn{2}{|c|}{ Sixteen Days } & \multicolumn{2}{|c|}{ Thirty-Two Days } \\
\hline & $\begin{array}{l}\text { \% Per } \\
\text { Organ }\end{array}$ & $\begin{array}{l}\% \text { Per } \\
\text { Gram }\end{array}$ & $\begin{array}{l}\text { \% Per } \\
\text { Organ }\end{array}$ & $\begin{array}{l}\% \text { Per } \\
\text { Gram }\end{array}$ & $\begin{array}{l}\text { \% Per } \\
\text { Organ }\end{array}$ & $\begin{array}{l}\text { \% Per } \\
\text { Gram }\end{array}$ & $\begin{array}{l}\% \text { Per } \\
\text { Organ }\end{array}$ & $\begin{array}{l}\text { \% Per } \\
\text { Gram }\end{array}$ \\
\hline Bone & 47.4 & 2.53 & 35.7 & 3.14 & 39.7 & 3.13 & 37.3 & 2.01 \\
\hline Liver & 29.2 & 3.27 & 29.3 & 4.07 & 10.3 & 1.45 & 4.30 & .45 \\
\hline Kidney & 2.59 & 1.35 & 2.06 & 1.24 & 1.16 & .68 & .57 & .25 \\
\hline Spleen & $<.07$ & $<.10$ & .07 & .14 & $<.03$ & $<.06$ & .08 & .13 \\
\hline
\end{tabular}

Average Recovery in Tissues of Administered Dose 107.\%

Rate of Elimination Equivalent to Half-Time in Body $\sim 40$ Days.

\section{ROUTE OF ADMINISTRATION - INTRAPULMONARY}

Values Indicate Distribution of Retained Activity

\begin{tabular}{|c|c|c|c|c|c|c|c|c|}
\hline & \multicolumn{2}{|c|}{ One Day } & \multicolumn{2}{|c|}{ Four Days } & \multicolumn{2}{|c|}{ Sixteen Days } & \multicolumn{2}{|c|}{ Thirty-Two Days } \\
\hline & $\begin{array}{l}\text { \% Per } \\
\text { Organ }\end{array}$ & $\begin{array}{l}\text { \% Per } \\
\text { Gram }\end{array}$ & $\begin{array}{l}\% \text { Per } \\
\text { Organ }\end{array}$ & $\begin{array}{l}\% \text { Per } \\
\text { Gram }\end{array}$ & $\begin{array}{l}\text { \% Per } \\
\text { Organ }\end{array}$ & $\begin{array}{l}\% \text { Per } \\
\text { Gram }\end{array}$ & $\begin{array}{l}\text { \% Per } \\
\text { Organ }\end{array}$ & $\begin{array}{l}\% \text { Per } \\
\text { Gram }\end{array}$ \\
\hline Bone & 18.0 & .90 & 22.2 & 1.84 & 50.0 & 2.33 & 59.9 & 3.06 \\
\hline Lungs & 58.7 & 57.6 & 48.3 & 40.3 & 21.9 & 17.6 & 9.32 & 5.90 \\
\hline Liver & 17.2 & 2.54 & 20.1 & 2.27 & $18: 2$ & 1.58 & 3.24 & .40 \\
\hline Kidney & 2.04 & 1.15 & 1.72 & .84 & 1.38 & .59 & .61 & .34 \\
\hline Spleen & $<.2$ & $<.5$ & .08 & .10 & .13 & .17 & $<.5$ & $<1.0$ \\
\hline
\end{tabular}

\section{ROUTE OF ADMINISTRATION - ORAL}

Absorption from Digestive Tract $<0.5 \%$

Total Number of Animals Used . . . . . . . . . . . . 27

Total Number of Tissue Assays . . . . . . . . . . . 500

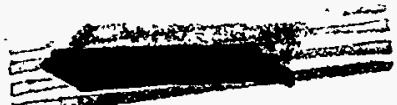


ELEMENT - NEPTUNIUM (Np $\left.{ }^{237}, \mathrm{~Np}^{238}\right)$ ADMINISTERED IN SOLUTION AS NpCl3

\section{ROUTE OF ADMINISTRATION - INTRAMUSCULAR}

Values Indicate Distribution of Absorbed Activity

Time in Days Following Administration

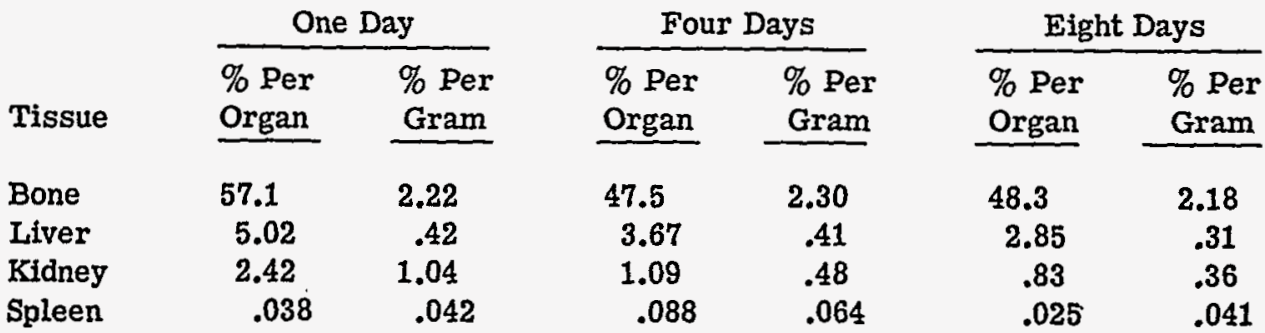

Average Recovery of Administered Dose in Tissues $114.7 \%$

Rate of Elimination Equivalent to Half-Time in Body $>15$ Days.

\section{ROUTE OF ADMINISTRATION - INTRAPULMONARY}

Values Indicate Distribution of Retained Activity

\begin{tabular}{|c|c|c|c|c|c|c|}
\hline & \multicolumn{2}{|c|}{ One Day } & \multicolumn{2}{|c|}{ Four Days } & \multicolumn{2}{|c|}{ Eight Days } \\
\hline$\cdot$ & $\begin{array}{l}\text { \% Per } \\
\text { Organ }\end{array}$ & $\begin{array}{l}\% \text { Per } \\
\text { Gram }\end{array}$ & $\begin{array}{l}\text { \% Per } \\
\text { Organ. }\end{array}$ & $\begin{array}{l}\% \text { Per } \\
\text { Gram }\end{array}$ & $\begin{array}{l}\% \text { Per } \\
\text { Organ }\end{array}$ & $\begin{array}{l}\text { \% Per } \\
\text { Gram }\end{array}$ \\
\hline Bone & 56.3 & 3.34 & 63.6 & 4.30 & 67.0 & 2.68 \\
\hline Lung & 18.6 & 9.07 & 13.4 & 7.78 & 9.75 & 5.74 \\
\hline Liver & 6.52 & .53 & 4.52 & .43 & 3.17 & .22 \\
\hline Kidney & 3.48 & 1.17 & 1.52 & .70 & .61 & .24 \\
\hline
\end{tabular}

\section{ROUTE OF ADMINLSTRATION - ORAL}

Absorption from Digestive Tract $<0.1 \%$

Total Number of Animals Used . . . . . . . . . . . . 21

Total Number of Tissue Assays . . . . . . . . . . . . 300

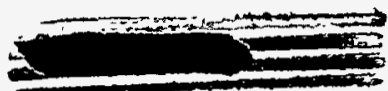

, sing 


\section{ELEMENT - URANIUM FISSION MIXTURE ADMINISTERED AS A SOLUTION OF THE CHLORIDES}

ROUTE OF ADMINISTERED - INTRAMUSCULAR

Values Indicate Distribution of Absorbed Activity

Time in Days Following Administration

\begin{tabular}{|c|c|c|c|c|c|c|}
\hline \multirow[b]{2}{*}{ Tissue } & \multicolumn{2}{|c|}{ Four Days } & \multicolumn{2}{|c|}{ Sixteen Days } & \multicolumn{2}{|c|}{ Sixty-Four Days } \\
\hline & $\begin{array}{l}\text { \% Per } \\
\text { Organ }\end{array}$ & $\begin{array}{l}\% \text { Per } \\
\text { Gram }\end{array}$ & $\begin{array}{l}\% \text { Per } \\
\text { Organ }\end{array}$ & $\begin{array}{l}\text { \% Per } \\
\text { Gram }\end{array}$ & $\begin{array}{l}\text { \% Per } \\
\text { Organ }\end{array}$ & $\begin{array}{l}\% \text { Per } \\
\text { Gram }\end{array}$ \\
\hline Bone & 48.9 & 2.27 & 45.9 & 1.74 & 39.7 & 1.21 \\
\hline Liver & 14.0 & 1.53 & 6.72 & .52 & 1.44 & .11 \\
\hline Kianey & 1.49 & .63 & .73 & .27 & .58 & .20 \\
\hline Spleen & .13 & .13 & .19 & .12 & .13 & .10 \\
\hline
\end{tabular}

Excretion Rate Equivalent to Half-Time in Body $\sim 80$ Days.

ROUTE OF ADMINISTRATION - ORAL (Absorption 5\% of

Administered Dose)

\begin{tabular}{|c|c|c|c|c|c|c|}
\hline & \multicolumn{2}{|c|}{ Four Days } & \multicolumn{2}{|c|}{ Sixteen Days } & \multicolumn{2}{|c|}{ Sixty-Four Days } \\
\hline & $\begin{array}{l}\% \text { Per } \\
\text { Organ }\end{array}$ & $\begin{array}{l}\% \text { Per } \\
\text { Gram }\end{array}$ & $\begin{array}{l}\text { \% Per } \\
\text { Organ }\end{array}$ & $\begin{array}{l}\% \text { Per } \\
\text { Gram }\end{array}$ & $\begin{array}{l}\% \text { Per } \\
\text { Organ }\end{array}$ & $\begin{array}{l}\% \text { Per } \\
\text { Gram }\end{array}$ \\
\hline Bone & 3,85 & .18 & 5.30 & .14 & 2.38 & .077 \\
\hline Liver & .030 & .0022 & .017 & .0023 & .011 & .0011 \\
\hline Kidney & .0066 & .0027 & .0030 & .0005 & .007 & .002 \\
\hline Spleen & .0066 & .0045 & .0030 & .0033 & .006 & .006 \\
\hline
\end{tabular}

Recovery of Administered Dose in Tissues for both routes of administration $103.9 \%$ Excretion Rate Equivalent to Half-Time in Body $\sim 80$ Days

ROUTE OF ADMINISTRATION - INTRAPULMONARY

\begin{tabular}{|c|c|c|c|c|c|c|}
\hline & \multicolumn{2}{|c|}{ Four Days } & \multicolumn{2}{|c|}{ Sixteen Days } & \multicolumn{2}{|c|}{ Sixty-Four Days } \\
\hline & $\begin{array}{l}\overline{\% \text { Per }} \\
\text { Organ }\end{array}$ & $\begin{array}{l}\% \text { Per } \\
\text { Gram }\end{array}$ & $\begin{array}{l}\text { \% Per } \\
\text { Organ }\end{array}$ & $\begin{array}{l}\% \text { Per } \\
\text { Gram }\end{array}$ & $\begin{array}{l}\% \text { Per } \\
\text { Organ }\end{array}$ & $\begin{array}{l}\% \text { Per } \\
\text { Gram }\end{array}$ \\
\hline Bone & 35.8 & 1.99 & 60.7 & 3.38 & 73.8 & 4.09 \\
\hline Lungs & 37.9 & 18.6 & 21.4 & 12.7 & 7.25 & 2.90 \\
\hline Liver & 5.57 & .36 & 7.43 & .63 & 3.49 & .22 \\
\hline Kidney & .59 & .19 & .74 & .28 & 1.00 & .31 \\
\hline Spleen & .05 & .04 & .12 & .12 & .24 & .15 \\
\hline
\end{tabular}

Total Number of Animals Used $\ldots \ldots \ldots \ldots$

Total Number of Tissues Assayed .............. 1,400

(4)

END OF DOCUMEN

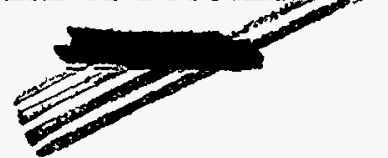




\section{DISCLAMMER}

Portions of this document may be illegible in electronic image products. Images are produced from the best available original document. 